\title{
Erratum: Positivity in the Sky: Constraining dark energy and modified gravity from the UV [Phys. Rev. D 101, 021502(R) (2020)]
}

\author{
S. Melville@ and J. Noller
}

(Received 29 July 2020; published 12 August 2020)

DOI: 10.1103/PhysRevD.102.049902

Here, we include a subleading correction to the positivity prior on the cosmological background (missing in the main text) and show that doing so only very mildly affects the computation of the resulting cosmological parameter constraints.

Positivity bounds: Expanding (3) in the main text about a flat background $\left(g_{\mu \nu}=\eta_{\mu \nu}+h_{\mu \nu} / M_{\mathrm{Pl}}\right)$, the tree-level amplitude for $\phi \phi \rightarrow \phi \phi$ takes the form (4), with

$$
\begin{aligned}
c_{s s t} & =-6\left(\bar{G}_{4, X X}+\bar{G}_{4, X}^{2} / \bar{G}_{4}\right), \\
c_{s s} & =2 \bar{G}_{2, X X}+4 \bar{G}_{4, X} \bar{G}_{2, X} / \bar{G}_{4},
\end{aligned}
$$

where an overbar indicates that the function is evaluated on the flat background $(\langle\phi\rangle=0)$. From (5) in the main text, the existence of a UV completion therefore requires

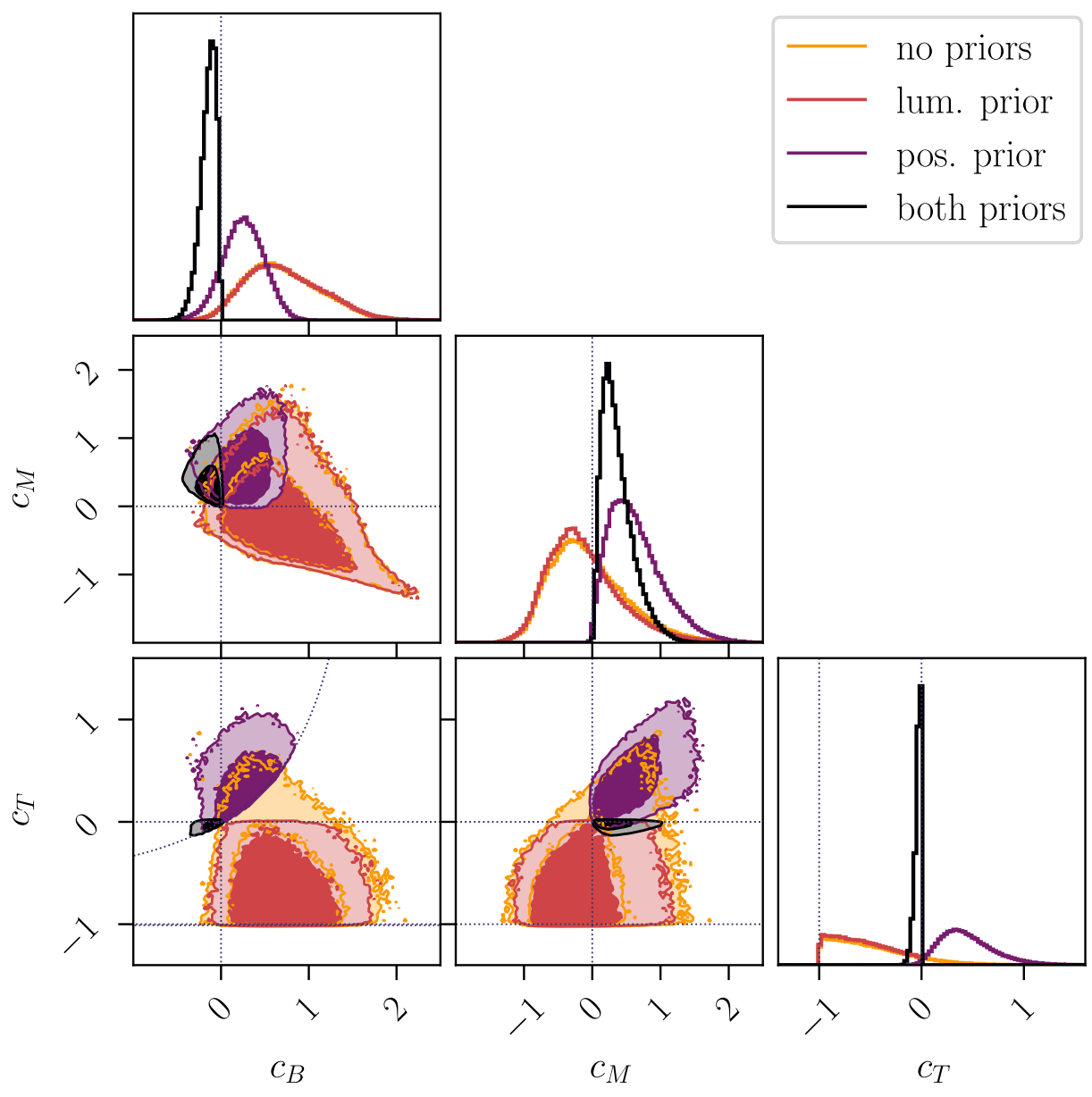

FIG. 1. Cosmological parameter constraints for the quartic Horndeski theory, as shown in Fig. 1 in the main text, but with the updated positivity prior derived from $\phi \phi \rightarrow \phi \phi$ scattering (A3). Overall constraints are only very mildly affected by this update. 
TABLE I. Posteriors on the dark energy/modified gravity $c_{i}$ parameters for the quartic Horndeski theory, as shown in Table 1 in the main text, but with the updated positivity prior derived from $\phi \phi \rightarrow \phi \phi$ scattering (A3). Overall constraints are only very mildly affected by this update.

\begin{tabular}{lccc}
\hline \hline & $c_{B}$ & $c_{M}$ & $c_{T}$ \\
\hline No priors & $0.71_{-0.71}^{+0.90}$ & $-0.02_{-0.89}^{+1.32}$ & $-1^{*} \leq c_{T}<0.25$ \\
Luminality prior & $0.73_{-0.72}^{+0.91}$ & $-0.09_{-0.84}^{+1.29}$ & $-1^{*} \leq c_{T} \leq 0^{*}$ \\
Positivity prior & $0.26_{-0.46}^{+0.46}$ & $0.67_{-0.58}^{+0.97}$ & $0.46_{-0.41}^{+0.64}$ \\
Both priors & $-0.16_{-0.22}^{+0.13}$ & $0.39_{-0.32}^{+0.60}$ & $-0.10<c_{T} \leq 0^{*}$ \\
\hline \hline
\end{tabular}

$$
2 \frac{\bar{G}_{4, X}}{\bar{G}_{4}} \geq-\frac{\bar{G}_{2, X X}}{\bar{G}_{2, X}}, \quad \frac{\bar{G}_{4, X}^{2}}{\bar{G}_{4}} \leq-\bar{G}_{4, X X},
$$

where we have assumed $\Lambda_{2} \gg \Lambda_{3}$. In the main text, $\bar{G}_{4}$ (and $\bar{G}_{2, X}$ ) were set to fixed constant values so that $\phi$ and $h_{\mu \nu}$ are "canonically normalized". However, when moving from the $\langle\phi\rangle=0$ background to the cosmological background (on which $\langle\phi\rangle$ is time dependent), there is inevitably a small time dependence introduced into $\bar{G}_{4}\left(\right.$ and $\left.\bar{G}_{2, X}\right)$. The purpose of this erratum is to include this time dependence in our positivity prior.

Linear cosmology and constraints: As before the main positivity-induced constraint on linear cosmology (for the quartic Horndeski model, we focus on in the main text) comes from the $c_{s s t}$ bound. In an expanding universe, it demands

$$
\text { pos.prior: } \alpha_{B} \leq \frac{2 \alpha_{T}}{1+\alpha_{T}} \text {, }
$$

where we have used that

$$
\alpha_{B}=2 \alpha_{T}+16 \frac{X^{2}}{M^{2}} G_{4, X X}, \quad \alpha_{M}=-\frac{1}{4} \frac{\dot{X}}{H X} \alpha_{B},
$$

as well as $M^{2}=2 G_{4} /\left(1+\alpha_{T}\right)$. In Fig. 1 and Table I, we show the constraints resulting from this updated bound. As a comparison with the previous plot and table in the main text shows, only very mild differences in the overall constraints result from the updated bound.

General Horndeski amplitude: For general Horndeski models beyond the specific example we focus on in the main text, the $\phi \phi \rightarrow \phi \phi$ amplitudes can be written in the form of Eq. (4) in the main text, with

$$
\begin{aligned}
c_{s s}= & 2\left(\bar{G}_{2, X X}-2 \bar{G}_{4, \phi \phi X}\right)-2 \frac{\bar{G}_{2, \phi \phi}}{\bar{G}_{2, X}}\left(\bar{G}_{3, X}+3 \bar{G}_{4, \phi X}\right)^{2} \\
& +4\left(\bar{G}_{2, \phi X}+\bar{G}_{3, \phi \phi}\right)\left(\bar{G}_{3, X}+3 \bar{G}_{4, \phi X}\right) \\
& +\frac{2}{\bar{G}_{4}}\left(2 \bar{G}_{4, X}-\bar{G}_{5, \phi}\right)\left(\bar{G}_{2, X}+\bar{G}_{3, \phi}-\bar{G}_{4, \phi \phi}\right) \\
c_{s s t}= & -2\left(3 \bar{G}_{4, X X}-\bar{G}_{5, \phi X}\right)+3\left(\bar{G}_{3, X}+3 \bar{G}_{4, \phi X}\right)^{2} \\
& -\frac{3}{2 \bar{G}_{4}}\left(2 \bar{G}_{4, X}-\bar{G}_{5, \phi}\right)^{2} .
\end{aligned}
$$

